\title{
Liderazgo transformacional y su impacto en la transferencia de conocimiento y el desempeño operativo en el contexto de la industria automotriz
}

DOI: https://doi.org/10.21158/01208160.n90.2021.2850

\author{
María Marisela Vargas-Salgado ${ }^{1}$ \\ Universidad Autónoma de Ciudad Juárez \\ mvargas021@yahoo.com.mx \\ Karla Gabriela Gómez-Bull ${ }^{2}$ \\ Universidad Autónoma de Ciudad Juárez \\ karla.gomez@uacj.mx
}

\section{Fecha de recepción: 09 de septiembre de 2019 \\ Fecha de aprobación: 05 de febrero de 2021 \\ Fecha de publicación: 26 de abril de 2021}

Cómo citar este artículo / To reference this article / Comment citer cet article / Para citar este artigo:

Vargas-Salgado, M. M.; Gómez-Bull, K. G. (2021). Liderazgo transformacional y su impacto en la transferencia de conocimiento y el desempeño operativo en el contexto de la industria automotriz. Revista Escuela de Administración de Negocios, (90), 11-26.

DOI: https://doi.org/10.21158/01208160.n90.2021.2850

\section{Resumen}

Actualmente las organizaciones se enfrentan a cambios retadores, con el fin de ser más competitivas. Los antecedentes teóricos revelan que el liderazgo es una variable necesaria en el contexto laboral, dado que a través de este se motiva y se guía a los seguidores a compartir conocimiento para realizar actividades que impacten considerablemente el desempeño y el éxito organizacional. Por tanto, el objetivo de este trabajo fue analizar el impacto que tiene el liderazgo transformacional sobre el desempeño operativo por medio de la mediación de la transferencia de conocimiento. Se utilizó un diseño cuantitativo de tipo no experimental, transversal, descriptivo y correlacional causal. La muestra fue no probabilística por conveniencia, considerándose como sujetos de estudio a los mandos medios que trabajan en la industria automotriz de la frontera norte de México; en total se encuestó a 300 participantes. Los datos descriptivos se analizaron mediante la herramienta estadística SPSS, mientras que la estadística inferencial se analizó a través de la herramienta modelado de ecuaciones estructurales, mediante la estimación de mínimos cuadrados parciales (PLS). Los resultados revelan que el liderazgo transformacional tiene un efecto positivo y significativo a un nivel de confianza del 99,9\% sobre la transferencia de conocimiento y el desempeño operativo.

Palabras clave: liderazgo transformacional; transferencia de conocimiento; desempeño operativo; desempeño organizacional; industria manufacturera; industria automotriz.

\footnotetext{
1 Doctora en Ciencias Administrativas - Universidad Autónoma de Ciudad Juárez, Chihuahua, México. Maestra en Administración Universidad Autónoma de Ciudad Juárez, Chihuahua, México. Licenciada en Administración de Empresas - Instituto Tecnológico de Parral, Chihuahua, México. ORCID: https://orcid.org/0000-0002-9670-5982

2 Ingeniero Industrial y de Sistemas - Universidad Autónoma de Ciudad Juárez. Maestra en Ingeniería Industrial - Universidad Autónoma de Ciudad Juárez. ORCID: https://orcid.org/0000-0002-6584-2597
} 


\title{
Transformational leadership and its impact on knowledge transfer and operational performance in the context of the automotive industry
}

\begin{abstract}
Today, organizations are facing challenging changes in pursuance of becoming more competitive. The theoretical background reveals that leadership is a necessary variable in the work context, since it motivates and guides followers to share knowledge in order to carry out activities that have a significant impact on organizational performance and success. Therefore, the aim of this work was to analyze the impact of transformational leadership on operational performance by means of the mediation of knowledge transfer. The study used a non-experimental, cross-sectional, descriptive and causal correlational quantitative design. The sample was non-probabilistic by convenience, considering middle managers working in the automotive industry in the northern border of Mexico as study subjects; a total of 300 participants were surveyed. Descriptive data were analyzed using the SPSS statistical tool, while inferential statistics were analyzed through the structural equation modeling tool, using the estimation of partial least squares (PLS). The results reveal that transformational leadership has a positive and significant effect on knowledge transfer and operational performance, at a $99.9 \%$ trust level.
\end{abstract}

Keywords: transformational leadership; knowledge transfer; operational performance; organizational performance; manufacturing industry; automotive industry.

\section{Liderança transformacional e seu impacto na transferência de conhecimento e desempenho operacional no contexto da indústria automotiva}

\section{Resumo}

Atualmente as organizações enfrentam mudanças desafiadoras com a finalidade de serem mais competitivas. Os antecedentes teóricos revelam que a liderança é uma variável necessária no contexto de trabalho, uma vez que por meio dela, os seguidores são motivados e orientados a compartilhar conhecimentos para a realização de atividades que impactam significativamente o desempenho e o sucesso organizacional. Portanto, o objetivo deste trabalho foi analisar o impacto que a liderança transformacional tem sobre o desempenho operacional, através da mediação da transferência de conhecimento. Foi utilizado um desenho quantitativo, não experimental, transversal, descritivo e causal correlacional. A amostra foi não probabilística por conveniência, considerando como sujeitos do estudo os gerentes de nível médio que trabalham na indústria automotiva da fronteira norte do México. No total, 300 participantes foram entrevistados. Os dados descritivos foram analisados por meio da ferramenta estatística SPSS, enquanto as estatísticas inferenciais foram analisadas por meio da ferramenta de modelagem de equações estruturais, através da estimativa de mínimos quadrados parciais (PLS). Os resultados revelam que a liderança transformacional tem um efeito positivo e significativo com um nível de confiança de $99,9 \%$ na transferência de conhecimento e no desempenho operacional.

Palavras-chave: liderança transformacional; transferência de conhecimento; desempenho operacional; desempenho organizacional; indústria transformadora; indústria automotiva. 


\section{Du leadership transformationnel et de sonimpact sur le transfert de connaissances et la performance opérationnelle dans le domaine de l'industrie automobile}

\section{Résumé}

Pour conserver leur compétitivité, les entreprises sont confrontées à des changements et des choix difficiles. Le contexte théorique révèle que le leadership est une variable fondamentale du domaine entrepreneurial dans la mesure où son rôle est de motiver les collaborateurs et de les inciter à partager leurs connaissances pour mener des activités ayant un impact significatif sur la performance et le succès de l'entreprise. L'objectif de cette étude est d'analyser l'impact du leadership transformationnel sur la performance opérationnelle à travers la médiation du transfert de connaissances. Une méthodologie quantitative, corrélationnelle, non expérimentale, transversale, descriptive et causale sera utilisée. L'échantillon n'est pas de type probabiliste et les 300 participants interrogés pour l'étude sont des cadres intermédiaires travaillant dans l'industrie automobile à la frontière nord du Mexique. Les données descriptives ont été analysées à l'aide de l'outil statistique SPSS alors que les statistiques inférentielles ont été analysées à l'aide d'un outil de modélisation d'équations structurelles utilisant l'estimation des moindres carrés partiels (PLS). Les résultats révèlent que le leadership transformationnel a un effet positif significatif et un niveau de confiance de $99,9 \%$ sur le transfert des connaissances et de la performance opérationnelle.

Mots-clés: leadership transformationnel; transfert de connaissances; performance opérationnelle; performance organisationnelle; industrie manufacturière; industrie automobile. 


\section{Introducción}

E n los últimos años las empresas han estado inmersas en constantes cambios, dado que operan en contextos dinámicos y competitivos (Al Zefeiti, 2017). En el ámbito organizacional es importante que los líderes desarrollen habilidades con la finalidad de lograr un desempeño eficiente en los trabajadores, dado que estos son parte significativa en la operación de la firma (OlivaAbusleme y Molina-Fuentes, 2016). Esto quiere decir que, las competencias laborales de cada empleado se ven reflejadas en los indicadores tanto individuales como organizacionales, ya que un empleado motivado está en mejores condiciones de alcanzar un buen desempeño (Cubillos-Rivera, Velásquez-Muriel y Reyes-Nova, 2014).

Cabe resaltar que las capacidades, las habilidades y las cualidades se consideran características individuales que tienen interacción con el trabajo y la organización, y que estas producen comportamientos y actitudes que, a su vez, tienen efecto sobre los resultados (Chiang, Méndez y Sánchez, 2010). En la misma vertiente, es importante entender que los recursos y las capacidades distintivos de cada persona permiten a las organizaciones tener un desempeño superior al de sus competidores (Ynzunza e Izar, 2013).

En las últimas décadas, la industria manufacturera de exportación mexicana se ha destacado por introducir mejores prácticas en los diversos sistemas de la organización y, en particular, en el sistema productivo. En este sentido, cabe resaltar que las empresas manufactureras cuentan con talento humano calificado. Además, este tipo de industria se encuentra en constante cambio con la finalidad de demostrar que es competitiva (Carrillo, 2014).

Dado que, por la naturaleza de su mercado, estas organizaciones se encuentran constantemente bajo presión por reducir sus costos, mejorar la calidad de sus productos y el servicio al cliente, ya que de esta forma se logra mantener su competitividad (Villa y Márquez, 2016), el objetivo de este trabajo de investigación fue analizar el impacto que tiene el liderazgo transformacional sobre el desempeño operativo, a través de la mediación de la transferencia de conocimiento en los empleados de mandos medios que laboran en la industria automotriz de la frontera norte.

Esta investigación se organizó en cuatro apartados: en el primero se abordan algunos antecedentes de liderazgo transformacional, transferencia de conocimiento y desempeño operativo; en el segundo se presenta el método de investigación; en el tercero se muestran los resultados y finalmente, se presentan la discusión y las conclusiones.

\section{Marco teórico}

\subsection{Liderazgo transformacional}

n el contexto organizacional, el liderazgo es una de las habilidades consideradas esenciales para quienes Cirigen y representan las firmas (Durán y Castañeda, 2015). El líder debe ser capaz de fortalecer la relación y la sinergia con los seguidores, a fin de lograr y mantener buenos resultados, los cuales se verán reflejados a partir del buen liderazgo que se ejerza en el ámbito laboral (Ahmad, Mohamed y Manaf, 2017). 
En particular, el liderazgo transformacional se considera uno de los más adecuados en el entorno organizacional, dado que, a través de este, las empresas promueven procesos favorables de cambio e innovación (Hermosilla et al., 2016). Además, los líderes logran impactar en gran medida en los seguidores y promueven así una visión a futuro a través del carisma y la potenciación de los seguidores (García-Guiu et al., 2016).

De acuerdo con la literatura es posible identificar cuatro componentes del liderazgo transformacional: a) el carisma, esto es, hacer referencia al valor, la identificación, el respeto y el orgullo que el líder es capaz de provocar en los seguidores; b) la inspiración, que hace referencia a la capacidad del líder para trasmitir la visión de forma optimista; c) la consideración individualizada, la cual implica la habilidad que posee el líder de identificar y atender necesidades particulares; y d) la estimulación intelectual, esto es, la forma en la que el líder enfrenta los problemas con miras a cumplir los objetivos (Avolio y Bass, 1995; Avolio, Bass y Jung, 1999; Menon, 2001).

Cabe resaltar que los líderes transformacionales influyen de forma considerable en los seguidores, dado que estos llevan a transformar las empresas en organizaciones más eficientes, con lo cual logran impactar en los objetivos organizacionales y alcanzar altos niveles de productividad. Así mismo, este estilo de liderazgo ayuda a innovar al lograr cambios favorables en el contexto laboral (Morales, Martínez y Montes, 2007), además, estimula a cambiar la cultura, los comportamientos y las actitudes de los empleados, a fin de contribuir a la eficiencia organizacional (Hoch, Bommer, Dulebohn y Wu, 2018).

\subsection{Transferencia de conocimiento}

En el ámbito laboral, la transferencia de conocimiento es parte del proceso de gestión, ya que a través de esta se identifica, capta, comparte y utiliza el conocimiento, el cual puede verse favorecido por la experiencia de otros (Argote y Ingram, 2000; Gómez, 2006). Además, se entiende como un flujo a lo largo de la empresa que puede asimilarse y convertirse en acciones de conocimiento (Wang, Chia-Lin, Jiang y Klein, 2007).
Esta variable también se conoce como el aprendizaje indirecto, el cual se adquiere de la experiencia de otros; su importancia radica en el impacto que tiene sobre la mejora del rendimiento y el desempeño de las unidades receptoras (Argote y Fahrenkopf, 2016). Se dice que la transferencia de conocimiento, en los grupos en los que una persona posee, será significativa sobre el rendimiento de estos grupos (Kane, Argote y Levine, 2005).

En las últimas décadas, las nuevas economías destacan que el conocimiento organizacional es la principal ventaja competitiva de las empresas, por tanto, la trasferencia es un proceso estratégicamente estructurado (Jasimuddin y Zhang, 2009). Dado que dentro de la empresa se estimula la creación y el desarrollo de nuevos conocimientos, además de incrementar las habilidades para innovar y ser más competitivos en los nuevos mercados cambiantes (Tsai, 2001).

Ahora bien, el conocimiento se transfiere cuando internamente se rota a los empleados en la organización, se cambian tareas y se integran herramientas o redes en las que el conocimiento puede estar presente a través de mecanismos tales como la educación, la capacitación y la comunicación (Argote y Fahrenkopf, 2016). No obstante, algunos hallazgos hacen mención a la falta de profundidad en algunos de sus aspectos y a la poca capacidad de absorber el conocimiento del personal, debido a la rutina de producción, a la disminución de la capacidad de aprendizaje debido a la falta de aceptación de nuevos conocimientos, o bien a no percibir que esta transferencia es valiosa (Herlina, Syarifudin y Kartika, 2019).

En el contexto organizacional los líderes son la parte central del proceso de administración efectiva del conocimiento, es decir, de la creación, la transferencia y el aprovechamiento de este (Bryant, 2003). En particular, el liderazgo transformacional tiene relación con la conducta de crear y compartir conocimiento (Durán y Castañeda, 2015; Song, Kolb, Lee y Kim, 2012). Uno de los beneficios más importantes de este tipo de liderazgo es que los seguidores incrementan sus oportunidades de solucionar problemas y tomar decisiones (Srivastava, Bartol y Locke, 2006). 
Se afirma que las características que identifican a los líderes transformacionales les permiten transferir conocimiento a los seguidores e impactar de forma considerable en la generación de nuevas ideas (Delgado-Almonte, Pedraja-Rejas y RodríguezPonce, 2010). De acuerdo con lo anterior, se plantea la siguiente hipótesis:

- H1: el liderazgo transformacional incide positiva y significativamente sobre la transferencia de conocimiento.

\subsection{Desempeño operativo}

Las empresas continuamente tienen el interés de mantener alineados los objetivos con las estrategias corporativas, con la finalidad de que los resultados se vean reflejados en los métricos organizacionales; cabe resaltar que esto es posible cuando se tiene un clima organizacional óptimo, además de contar con empleados comprometidos y motivados (CubillosRivera et al., 2014). El desempeño compete tanto al trabajador como a la empresa; pues además de producir beneficios a los empleados, puede contribuir a mejorar la productividad de la empresa y con esto a su rentabilidad.

No obstante, el desempeño se puede ver afectado por el clima organizacional, por lo cual es importante que las organizaciones mantengan un ambiente laboral adecuado que contribuya a obtener mejores resultados en la productividad y en los objetivos organizacionales (Cubillos-Rivera et al., 2014). En ese sentido el desempeño operativo se verá reflejado en la ejecución de las operaciones realizadas día a día tanto en la parte productiva como en la administrativa (de Leeuw y van den Berg, 2011; Inman, Sale, Green Jr. y Whitten, 2011).

En el desempeño organizacional se busca una evaluación holística del cumplimiento de los objetivos y las metas en función de todos los involucrados en la organización (Machorro et al., 2016). Cabe destacar que existen diversos criterios para estimar y evaluar el desempeño de los empleados de una organización, algunos de los cuales son: cantidad y calidad de trabajo, cooperación, responsabilidad, conocimiento del trabajo, asistencia y necesidad de supervisión, entre otros (Chiang et al., 2010).

Un aspecto fundamental en el proceso de desarrollo de personal de alto desempeño es lograr una diferenciación en el liderazgo. Esto es necesario, ya que cada trabajador deberá responder por sus actividades, las cuales se basan en la confianza compartida hacia el éxito tanto individual como grupal, además de que este deberá reflejarse en el exterior de la organización (Uribe et al., 2013). Vale la pena destacar que la competitividad obliga a las empresas a adoptar mejores prácticas que contribuyan al desempeño a través del costo, la calidad, el tiempo de entrega y la flexibilidad operacional (Inman et al., 2011).

El liderazgo transformacional se vincula favorablemente con el desempeño de los trabajadores, dado que los líderes enfrentan diversas dificultades, con el propósito de lograr los objetivos trazados a partir de las tareas que cada uno de los empleados realiza en su contexto laboral (Al Zefeiti, 2017). Es por ello que este tipo de líder impacta en gran medida en el desempeño operativo de los trabajadores (Carmeli, Gelbard y Reiter-Palmon, 2013). Por lo antes expuesto, se postula la siguiente hipótesis:

- H2: el liderazgo transformacional tiene un efecto positivo y significativo sobre el desempeño operativo.

Además, en las organizaciones es necesario propiciar el desarrollo de las competencias, las habilidades, la experiencia y los atributos de los trabajadores que son parte importante para la transferencia y el uso del conocimiento adquirido en relación con el desempeño (Paz, 2017), ya que la transferencia de conocimiento tiene una influencia significativa sobre el desempeño operativo de los trabajadores (Srivastava et al., 2006).

Al respecto, Carmeli, Gelbard y Reiter-Palmon (2013) resaltan que a través del liderazgo transformacional se facilita la transferencia de conocimiento interna y externa, así como la capacidad creativa para la solución de problemas entre los empleados, de forma 
que también impacta de manera considerable en el desempeño de los trabajadores. Por tanto, se plantea la siguiente hipótesis:

- H3: la transferencia de conocimiento influye positiva y significativamente sobre el desempeño operativo.

\section{Método}

E ste trabajo de investigación es de tipo cuantitativo, no experimental, transversal, descriptivo y correlacional causal. La muestra fue no probabilística, pues se consideró como sujetos de estudio a empleados que laboran como mandos medios en la industria maquiladora de exportación, específicamente en el sector automotriz; en total se obtuvieron 300 cuestionarios, los cuales fueron aplicados de forma directa a cada uno de los participantes en el entorno laboral.

Losítems del cuestionario se valoraron con una escala tipo Likert de cinco puntos de asignación de respuesta para liderazgo transformacional y transferencia de conocimiento, la escala fue: nunca, raras veces, algunas veces, muchas veces y siempre. El liderazgo transformacional se midió con 17 ítems, a través de la escala validada por Vargas-Salgado et al. (2016). Por su parte, la transferencia de conocimiento se valoró con cinco ítems (Máynez, 2011). Finalmente, el desempeño operativo se evaluó con un instrumento de ocho ítems, construido a partir de los ya existentes (Bowersox et al., 2000; Boyer y Lewis, 2002; Chang et al., 2003; Ward y Duray, 2000).

Con respecto a los indicadores operativos de la empresa, para los primeros tres ítems, la escala fue: totalmente en desacuerdo, en desacuerdo, ni de acuerdo ni en desacuerdo, de acuerdo, totalmente de acuerdo; para el resto de los ítems en relación con el desempeño operativo, tomando como base de comparación los competidores en la misma industria, la escala fue: muy pobre por debajo de la industria, inferior a la competencia, equivalente a la competencia, mejor que el promedio de competidores y muy superior a la competencia. La estadística descriptiva se analizó mediante el software SPSS versión 21, mientras que la estadística inferencial a través de la herramienta Modelo de Ecuaciones Estructurales, mediante la estimación de mínimos cuadrados parciales (PLS). El modelo se corrió con el software SmartPLS3.

\section{Resultados}

$\mathrm{E}^{\mathrm{n}}$ la tabla 1 se presentan los resultados demográficos referentes al perfil de los sujetos de estudio y de los puestos de trabajo. Se muestra que casi el $64 \%$ de los participantes son hombres, mientras que el $70 \%$ tiene entre 20 y 39 años. El $85 \%$ cuenta con una carrera profesional terminada y alrededor del $88 \%$ de los sujetos de estudio tienen más de un año de antigüedad en la organización.
Por otra parte, con respecto al perfil de los puestos, los datos revelan que el 53,4 \% laboran en los departamentos de ingeniería y administración, mientras que en el puesto que ocupan los sujetos de estudio se destaca el de ingeniero con un 54,7\% y el de supervisor con un 30,3\%. De acuerdo con el perfil de las organizaciones, el $100 \%$ corresponde a la industria automotriz. 
Tabla 1. Perfil de los sujetos de estudio y de los puestos de trabajo $\mathrm{n}=300$

\begin{tabular}{|c|c|c|c|c|c|}
\hline Características & $\begin{array}{l}\text { Número de } \\
\text { personas }\end{array}$ & $\%$ & Características & $\begin{array}{l}\text { Número de } \\
\text { personas }\end{array}$ & $\%$ \\
\hline Sexo & & & Departamento & & \\
\hline Hombre & 191 & 63,6 & Ingeniería & 113 & 37,7 \\
\hline Mujer & 109 & 36,4 & Administrativo & 47 & 15,7 \\
\hline Edad & & & Calidad & 40 & 13,3 \\
\hline Menos de 20 & 0 & 0,0 & Producción & 35 & 11,7 \\
\hline Entre 20 y 29 años & 119 & 39,7 & Materiales & 33 & 11,0 \\
\hline Entre 30 y 39 años & 91 & 30,3 & Mantenimiento & 19 & 6,3 \\
\hline Entre 40 y 49 años & 60 & 20,0 & Otros & 13 & 4,3 \\
\hline Entre 50 y 59 & 28 & 9,34 & & & \\
\hline Mas de 60 & 2 & 0,66 & & & \\
\hline Antigüedad & & & Puesto & & \\
\hline Menos de un año & 35 & 11,7 & Gerente & 21 & 7,0 \\
\hline $\begin{array}{l}\text { Entre uno y tres } \\
\text { años }\end{array}$ & 122 & 40,7 & Supervisor & 91 & 30,3 \\
\hline $\begin{array}{l}\text { Entre cuatro y siete } \\
\text { años }\end{array}$ & 73 & 24,3 & Ingeniero & 164 & 54,7 \\
\hline Más de ocho años & 70 & 23,3 & Otro & 24 & 8,0 \\
\hline \multicolumn{6}{|l|}{ Escolaridad } \\
\hline $\begin{array}{l}\text { Preparatoria } \\
\text { terminada }\end{array}$ & 9 & 3,0 & & & \\
\hline $\begin{array}{l}\text { Carrera profesional } \\
\text { no terminada }\end{array}$ & 36 & 12,0 & & & \\
\hline $\begin{array}{l}\text { Carrera profesional } \\
\text { terminada }\end{array}$ & 197 & 65,7 & & & \\
\hline Maestría & 58 & 19,3 & & & \\
\hline
\end{tabular}

Fuente. Elaboración propia a partir de datos de SPSS.

En el modelo de ecuaciones estructurales se valoró la validez convergente y la validez discriminante. En la primera, se evaluó la fiabilidad compuesta, el alfa de Cronbach y el análisis de varianza extraída (véase la Tabla 2). Se puede observar que la mayoría de los ítems exhiben cargas aceptables, dado que son mayores a 0,70 , lo cual indica que existe mayor varianza compartida entre el constructo y las variables latentes (Bagozzi y Fornell, 1982; Hulland, 1999). Aunque no para los ítems D1 - 0,565-, D2 -0,670—, D3 -0,638-, L1 -0,655- y L16 - 0,697 - , los cuales revelan valores menores a 0,70 ; pero, puesto que estos cumplen con una carga mayor a 0,50, se consideran aceptables dado que en el constructo existen otros indicadores que presentan cargas altas (Chin, 1998). Con respe cto a los valores $t$ se puede apreciar que estos se encuentran en un rango de 11,140 a 56,754, por lo que puede afirmarse que todas las cargas resultaron estadísticamente significativas — valores $t$ significativos al 99,9 \%, ya que estos son superiores a 3,291-.

La fiabilidad comúnmente se mide a través del alfa de Cronbach. Se recomienda que este indicador exhiba valores superiores a 0,70 , lo cual muestra que los indicadores son consistentes en su medida (Fornell y 
Bookstein, 1982; Hair et al., 2007; Oviedo y CampoArias, 2005). Los resultados de cada uno de los constructos cumplen con el criterio señalado, dado que superan el punto de corte: desempeño operativo $-0,880$-, liderazgo transformacional $-0,954$ transferencia de conocimiento $-0,959-$.

Por otra parte, la fiabilidad compuesta se considera una mejor medida de consistencia interna, la cual representa el grado en el cual cada una de las variables latentes indican al constructo (Hair et al., 2007; Máynez, 2011). Los resultados de fiabilidad compuesta de cada una de las variables latentes exceden el punto de corte: desempeño operativo - 0,904-, liderazgo transformacional $-0,958$-, transferencia de conocimiento -0,926- En todos los casos los indicadores exceden el punto de corte de 0,70 .
Con respecto al análisis de varianza extraída AVE por sus siglas en inglés para Average Variance Extracted-, se calcula la cantidad de varianza que un constructo obtiene a través de sus indicadores, respecto a la varianza del error de medida (Chin, 1998); se recomienda que el indicador de la varianza extraída sea superior a 0,50 , lo cual implica que más del $50 \%$ de la varianza se explica mediante los indicadores de las variables latentes (Chin, 1998; Fornell y Bookstein, 1982; Gefen y Straub, 2005).

Los resultados revelados en la tabla 3 evidencian que también en este criterio se exceden los puntos de corte: desempeño operativo $-0,543-$, liderazgo transformacional $-0,575-$ y transferencia de conocimiento $-0,714-$. Se puede afirmar que existe validez convergente, dado que los indicadores del alfa de Cronbach, fiabilidad compuesta y análisis de varianza extraída exceden los puntos de corte.

Tabla 2. Indicadores del modelo de medida

\begin{tabular}{|c|c|c|c|c|c|}
\hline Ítem & Cargas & Valor $t$ & $\begin{array}{l}\text { Fiabilidad } \\
\text { compuesta }\end{array}$ & $\begin{array}{l}\text { Alfa de } \\
\text { Cronbach }\end{array}$ & $\begin{array}{c}\text { Análisis de varianza } \\
\text { extraída AVE }\end{array}$ \\
\hline \multicolumn{6}{|l|}{ Desempeño operativo } \\
\hline $\begin{array}{l}\text { D1 Nuestro costo de producción es menor que } \\
\text { el de la competencia }\end{array}$ & 0,565 & $11,140^{* * *}$ & \multirow{8}{*}{0,904} & \multirow{8}{*}{0,880} & \multirow{8}{*}{0,543} \\
\hline $\begin{array}{l}\text { D2 Nuestro volumen de desperdicio interno } \\
\text { — scrap — es menor que el de la competencia }\end{array}$ & 0,670 & $16,131^{* * *}$ & & & \\
\hline $\begin{array}{l}\text { D3 Nuestro volumen de retrabajo es menor que } \\
\text { el de la competencia }\end{array}$ & 0,638 & $13,163^{* * *}$ & & & \\
\hline $\begin{array}{l}\text { D4 Cumplimiento a las especificaciones de la } \\
\text { calidad del producto }\end{array}$ & 0,757 & $26,814^{* * *}$ & & & \\
\hline D5 Desempeño del tiempo de entrega & 0,803 & $32,284^{* * *}$ & & & \\
\hline $\begin{array}{l}\text { D6 Flexibilidad para cambiar la mezcla de } \\
\text { productos }\end{array}$ & 0,805 & $35,581^{* * *}$ & & & \\
\hline $\begin{array}{l}\text { D7 Flexibilidad para cambiar el volumen de } \\
\text { producción }\end{array}$ & 0,835 & $38,936^{* * *}$ & & & \\
\hline $\begin{array}{l}\text { D8 Velocidad en la producción para introducir } \\
\text { nuevos productos }\end{array}$ & 0,779 & $26,218^{* * *}$ & & & \\
\hline
\end{tabular}




\begin{tabular}{|c|c|c|c|c|c|}
\hline \multicolumn{6}{|l|}{ Liderazgo transformacional } \\
\hline $\begin{array}{l}\text { L1 Al tomar decisiones, siempre toma en cuenta } \\
\text { las consecuencias de estas }\end{array}$ & 0,655 & $17,412^{* * *}$ & \multirow{17}{*}{0,958} & \multirow{17}{*}{0,954} & \multirow{17}{*}{0,575} \\
\hline $\begin{array}{l}\text { L10 Me sugiere nuevas formas de hacer mi } \\
\text { trabajo }\end{array}$ & 0,725 & $20,374^{* * *}$ & & & \\
\hline $\begin{array}{l}\text { L11 Me ayuda a ver los problemas desde } \\
\text { diferentes puntos de vista }\end{array}$ & 0,805 & $34,006^{* * *}$ & & & \\
\hline $\begin{array}{l}\text { L12 Me sugiere considerar nuevas y distintas } \\
\text { perspectivas cuando resuelvo problemas }\end{array}$ & 0,786 & $29,927^{* * *}$ & & & \\
\hline $\begin{array}{l}\text { L13 Me estimula a expresar mis ideas y } \\
\text { opiniones }\end{array}$ & 0,793 & $38,309^{* * *}$ & & & \\
\hline $\begin{array}{l}\text { L14 Dedica tiempo a enseñarme cómo realizar } \\
\text { mi trabajo }\end{array}$ & 0,748 & $25,909^{* * *}$ & & & \\
\hline $\begin{array}{l}\text { L15 Dedica tiempo en orientarme para que } \\
\text { realice mejor mi trabajo }\end{array}$ & 0,754 & $27,365^{* * *}$ & & & \\
\hline L16 Toma en cuenta mis necesidades personales & 0,697 & $19,018^{* * *}$ & & & \\
\hline $\begin{array}{l}\text { L17 Me asigna proyectos significativos para } \\
\text { incrementar mi desarrollo personal }\end{array}$ & 0,707 & $18,708^{* * *}$ & & & \\
\hline L2 Es un modelo a seguir & 0,750 & $30,751^{* * *}$ & & & \\
\hline $\begin{array}{l}\text { L3 Actúa de modo que se gana el respeto de los } \\
\text { demás }\end{array}$ & 0,735 & $25,714^{* * *}$ & & & \\
\hline $\begin{array}{l}\text { L4 Hace que me sienta orgulloso de pertenecer } \\
\text { al equipo de trabajo }\end{array}$ & 0,772 & $30,973^{* * *}$ & & & \\
\hline $\begin{array}{l}\text { L5 Logra que tenga confianza en mis juicios y } \\
\text { mis decisiones }\end{array}$ & 0,804 & $33,842^{* * *}$ & & & \\
\hline $\begin{array}{l}\text { L6 Logra que me comprometa con la visión a } \\
\text { futuro }\end{array}$ & 0,812 & $40,198^{* * *}$ & & & \\
\hline L7 Muestra el futuro de forma optimista & 0,752 & $25,571^{* * *}$ & & & \\
\hline $\begin{array}{l}\text { L8 Tiende a hablar con entusiasmo sobre las } \\
\text { metas }\end{array}$ & 0,787 & $27,933^{* * *}$ & & & \\
\hline $\begin{array}{l}\text { L9 Me ayuda a ver los beneficios que tendré si } \\
\text { alcanzo las metas organizacionales }\end{array}$ & 0,794 & $33,315^{* * *}$ & & & \\
\hline \multicolumn{6}{|l|}{ Transferencia de conocimiento } \\
\hline $\begin{array}{l}\text { TC1 Es común que los empleados compartan } \\
\text { sus conocimientos }\end{array}$ & 0,781 & $26,342^{* * *}$ & \multirow{5}{*}{0,926} & \multirow{5}{*}{0,900} & \multirow{5}{*}{0,714} \\
\hline $\begin{array}{l}\text { TC2 Gracias a los conocimientos compartidos, } \\
\text { las actividades laborales son cada vez más } \\
\text { eficientes }\end{array}$ & 0,876 & $56,754^{* * *}$ & & & \\
\hline $\begin{array}{l}\text { TC3 Tomando como base los conocimientos } \\
\text { compartidos, realizamos cambios en nuestra } \\
\text { forma de trabajar }\end{array}$ & 0,856 & $46,815^{* * *}$ & & & \\
\hline $\begin{array}{l}\text { TC4 Los conocimientos compartidos se vuelven } \\
\text { parte de las rutinas normales de trabajo }\end{array}$ & 0,851 & $46,259^{* * *}$ & & & \\
\hline $\begin{array}{l}\text { TC } 5 \text { Al compartir conocimiento somos capaces } \\
\text { de crear nuevas prácticas laborales }\end{array}$ & 0,858 & $41,163^{* * *}$ & & & \\
\hline
\end{tabular}

*** Significativo con un alpha de 0,001, si t > 3,291, para una prueba de dos colas

Fuente. Elaboración propia a partir de resultados de SmartPLS 3. 
Con respecto a la validez discriminante, esta indica en qué medida un constructo es diferente de otros en el mismo modelo (Bagozzi y Fornell, 1982). Además, muestra cuando cada uno de los elementos de un constructo se correlaciona débilmente con el resto (Gefen y Straub, 2005). La validez discriminante se puede determinar a través del criterio de la varianza extraída (AVE) y el Heterotrait-Monotrait Ratio (HTMT).

En referencia al primer criterio, este tipo de validez se determina calculando la varianza compartida entre el constructo y el resto de las variables latentes del modelo de medición, además, se explora si la varianza extraída es mayor que la varianza compartida (Fornell y Bookstein, 1982). En la tabla 3 se exhibe la matriz de correlaciones entre los constructos, en la cual, en la diagonal, se aprecia que la raíz cuadrada de la varianza extraída es mayor que la varianza compartida, por lo cual se puede afirmar que existe validez discriminante.

Tabla 3. Validez discriminante de acuerdo al criterio (AVE)

\begin{tabular}{|l|c|c|c|}
\hline \multicolumn{1}{|c|}{ Constructo } & $\begin{array}{c}\text { Desempeño } \\
\text { operativo }\end{array}$ & $\begin{array}{c}\text { Liderazgo } \\
\text { transformacional }\end{array}$ & $\begin{array}{c}\text { Transferencia de } \\
\text { conocimiento }\end{array}$ \\
\hline Desempeño operativo & $\mathbf{0 , 7 3 7}$ & & \\
\hline Liderazgo transformacional & 0,467 & 0,759 & 0,845 \\
\hline $\begin{array}{l}\text { Transferencia de } \\
\text { conocimiento }\end{array}$ & 0,516 & 0,464 & \\
\hline
\end{tabular}

Fuente. Elaboración propia a partir de resultados de SmartPLS 3.

Con respecto al criterio Heterotrait-Monotrait Ratio (HTMT), utiliza el promedio de las correlaciones heterotrait-heteromethod, el mismo que evalúa las correlaciones entre los indicadores que valoran constructos distintos. La validez discriminante puede establecerse como criterio o como prueba estadística, en este trabajo de investigación se analiza como criterio y usualmente el punto de corte es 0,85 o 0,90, el mismo que se contrasta con los heterotrait-monotrait ratios, los cuales deben de ser menores que el punto de corte. En la tabla 4 se exhiben los indicadores que presentan valores menores al punto de corte, por lo cual se puede confirmar que existe validez discriminante (Henseler, Ringle y Sarstedt, 2015).

Tabla 4. Validez discriminante de acuerdo al Heterotrait-Monotrait Ratio (HTMT)

\begin{tabular}{|l|c|c|}
\hline \multicolumn{1}{|c|}{ Constructo } & Desempeño operativo & Liderazgo transformacional \\
\hline Desempeño operativo & & \\
\hline Liderazgo transformacional & 0,503 & \\
\hline Transferencia de conocimiento & 0,547 & 0,484 \\
\hline
\end{tabular}

Fuente. Elaboración propia a partir de resultados de SmartPLS 3.

En la figura 1 se muestran los coeficientes de los tres paths planteados, en los cuales los tres cumplen con el valor mínimo aceptable de 0,20: liderazgo transformacional $\rightarrow$ desempeño operativo $-0,291$-, liderazgo transformacional $\rightarrow$ transferencia de conocimiento $-0,464-$ y transferencia de conocimiento $\rightarrow$ desempeño operativo - 0,381 - ; en los tres casos el valor $t$ es superior a 3,291, por lo cual se puede afirmar que los valores son fiables, es decir, estadísticamente significativos con un nivel de confianza del 99,9\%. 
En lo que respecta al coeficiente $\mathrm{r}^{2}$, este indica que las variables endógenas reciben influencia explicativa de otra variable predictora, en el cual, de forma moderada, el liderazgo transformacional predice a la transferencia de conocimiento - 0,215- y el liderazgo transformacional, conjuntamente con la transferencia de conocimiento, predicen el desempeño operativo $-0,33-$.

Figura 1. Modelo estructural

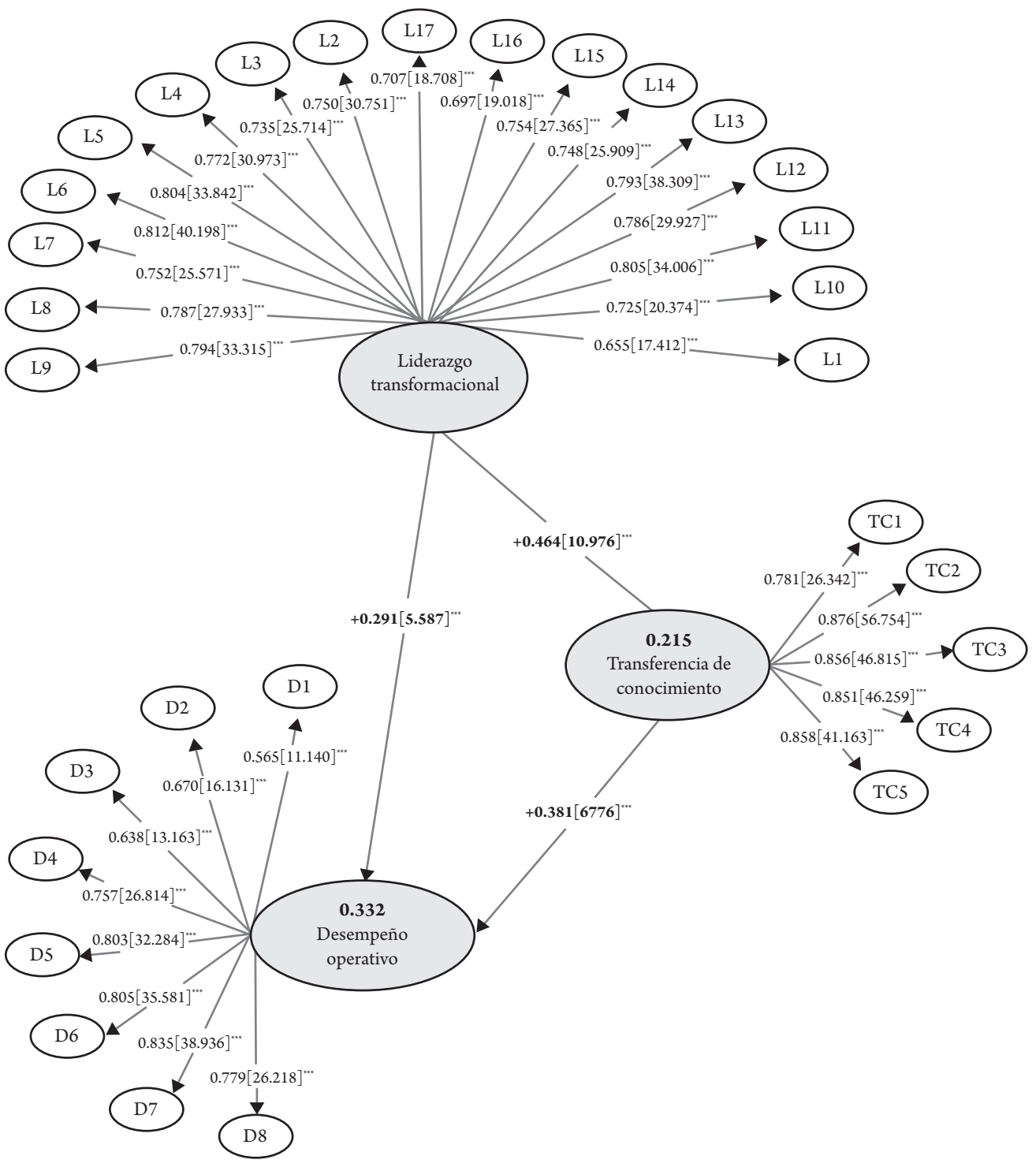

*** Significativo con un alpha de 0,001, si $\mathrm{t}>3,291$, para una prueba de dos colas

Fuente. Elaboración propia a partir de resultados de Smart PLS.

Los resultados obtenidos con respecto a las hipótesis planteadas, revelados en la tabla 5, permiten afirmar que estas son estadísticamente significativas a un nivel de confianza del 99,9\% — alpha del 0,001—, ya que sus valores $t$ son superiores a 3,291, donde: $\mathrm{H} 1-t=10,976-, \mathrm{H} 2-t=5,587-\mathrm{y} \mathrm{H} 3-t=6,776]$. 
Por tanto, no se rechaza ninguna de las hipótesis planteadas, dado que es posible reafirmar que el liderazgo transformacional incide positiva y significativamente sobre la transferencia de conocimiento. El liderazgo transformacional tiene un efecto positivo y significativo sobre el desempeño operativo y la transferencia de conocimiento influye positiva y significativamente sobre el desempeño operativo.

Tabla 5. Prueba de hipótesis

\begin{tabular}{|l|c|c|c|}
\hline \multicolumn{1}{|c|}{ Hipótesis } & Coeficiente path & Valor $\boldsymbol{t}$ & Resultado \\
\hline $\begin{array}{l}\text { H1. El liderazgo transformacional incide positiva y } \\
\text { significativamente sobre la transferencia de conocimiento. }\end{array}$ & 0,464 & $10,976^{* * *}$ & No rechazo \\
\hline $\begin{array}{l}\text { H2. El liderazgo transformacional tiene un efecto positivo y } \\
\text { significativo sobre el desempeño operativo. }\end{array}$ & 0,291 & $5,587^{* * *}$ & No rechazo \\
\hline $\begin{array}{l}\text { H3. La transferencia de conocimiento influye positiva y } \\
\text { significativamente sobre el desempeño operativo. }\end{array}$ & 0,381 & $6,776^{* * *}$ & No rechazo \\
\hline
\end{tabular}

*** Significativo con un alpha de 0,001, si t > 3,291, para una prueba de dos colas

Fuente. Elaboración propia.

\section{Discusión}

$\mathrm{E}^{\mathrm{s}}$ n el contexto de la industria manufacturera de exportación, esta investigación tuvo como objetivo analizar el impacto que tiene el liderazgo transformacional sobre el desempeño operativo, a través de la mediación de la transferencia de conocimiento en los empleados de mandos medios que laboran en la industria automotriz de la frontera norte. Los resultados revelan que en este contexto las relaciones son significativas, se reafirma que el liderazgo transformacional influye de forma significativa y directa sobre el desempeño operativo, y de forma indirecta a través de la mediación de la transferencia de conocimiento.

De acuerdo con los resultados, se afirma que el constructo de liderazgo transformacional es de gran relevancia en el contexto estudiado, pues es claro que este tipo de líder coadyuva en gran medida en los objetivos organizacionales y que, además, este constructo impacta significativamente sobre la transferencia de conocimiento de los empleados.

Los resultados coinciden con trabajos teóricos previos (Delgado-Almonte, Pedraja-Rejas y Rodríguez-Ponce, 2010; Durán y Castañeda, 2015; Song et al., 2012) que señalan cómo los líderes se preocupan por compartir y transferir conocimiento con los seguidores, con la finalidad de generar nuevas ideas en el ámbito estudiado. Es decir, que gracias a los conocimientos compartidos por parte de los trabajadores las actividades cotidianas son más eficientes, además, esto permite que los empleados realicen cambios en la forma de trabajar, ya que compartir conocimiento se considera una parte esencial de la rutina laboral.

En relación con la influencia del liderazgo transformacional sobre el desempeño operativo, los resultados muestran también resultados significativos, es decir, los líderes se enfrentan a diversas situaciones organizacionales, con la finalidad de que los trabajadores puedan lograr las metas proyectadas a partir de las actividades que desarrollan en el contexto laboral (Al Zefeiti, 2017). Esto es, los sujetos perciben que tienen un buen desempeño, dado que las entregas se cristalizan a tiempo, además consideran que en el contexto automotriz existe flexibilidad para realizar cambios en la mezcla de productos y cambios en el volumen de producción, sin afectar el desempeño de la organización. Cabe resaltar que tanto el líder 
como los seguidores están comprometidos con la visión a futuro, además de que el líder brinda la confianza que le permite a los empleados tomar decisiones con respecto al trabajo que realizan.

Referente a la influencia de la transferencia de conocimiento sobre el desempeño operativo, los resultados revelan que la transferencia de conocimiento incide fuertemente en el desempeño operativo. Es decir, en la industria manufacturera de exportación, los líderes enfatizan en el desarrollo de competencias y habilidades, dado que esto es importante para que los mandos medios de este entorno usen el conocimiento y lo transfieran, y este se vea reflejado a su vez en el desempeño de las actividades (Paz, 2017).

\section{Conclusiones}

os hallazgos indican que, en el sector automo-
triz de la frontera norte de México, el liderazgo transformacional influye favorablemente en el desempeño operativo, a través de la mediación de la transferencia de conocimiento en los empleados de mandos medios. En este entorno de industria el líder transformacional refuerza en los seguidores la visión de los problemas desde diferentes puntos de vista, así como el panorama de los beneficios que tendrán si alcanzan los metas organizacionales. Además, a los empleados les gusta compartir sus conocimientos, ya que a través de este renuevan y mejoran las prácticas laborales.

Por lo tanto, es común ver que las empresas automotrices están orientadas a diseñar estrategias de manufactura que les permitan lograr un destacado desempeño basado en el tiempo, la entrega y la flexibilidad operacional, y con esto lograr ventajas competitivas en un contexto globalizado.

Por último, este trabajo tiene sus limitantes. Si bien cabe destacar que esta investigación contribuye con evidencia empírica al contexto estudiado, no se pueden generalizar los resultados, dado que es un estudio transversal, con una muestra no probabilística y realizado en la industria automotriz. Se recomienda en futuros estudios considerar contextos diferentes y estudios longitudinales, además de vincular los constructos estudiados con otras variables - tales como antecedentes o consecuentes - que permitan avanzar en el conocimiento y el éxito organizacional.

\section{Referencias}

Ahmad, R. B.; Mohamed, A. M. b.; Manaf, H. B. A. (2017). The relationship between transformational leadership characteristic and succession planning program in the Malaysian public sector. International Journal of Asian Social Science, 7(1), 19-30. DOI: https://doi.org/10.18488/ journal.1/2017.7.1/1.1.19.30

Al Zefeiti, S. (2017). The influence of transformational leadership behaviours on Oman public employees' work performance. Asian Social Science, 13(3), 102-116. DOI: https://doi. org/10.5539/ass.v13n3p102

Argote, L.; Fahrenkopf, E. (2016). Knowledge transfer in organizations: the roles of members, tasks, tools, and networks. Organizational Behavior and Human Decision Processes, 136, 146-159. DOI: https://doi.org/10.1016/j. obhdp.2016.08.003

Argote, L.; Ingram, P. (2000). Knowledge transfer: a basis for competitive advantage in firms. Organizational Behavior and Human Decision Processes, 82(1), 150-169. DOI: https://doi. org/10.1006/obhd.2000.2893

Avolio, B. J.; Bass, B. M. (1995). Individual consideration viewed at multiple levels of analysis: a multi-level framework for examining the diffusion of transformational leadership. Leadership Quarterly, 6(2), 199-218. DOI: https://doi. org/10.1016/1048-9843(95)90035-7 
Avolio, B. J.; Bass, B. M.; Jung, D. I. (1999). Re-examining the components of transformational and transactional leadership using the Multifactor Leadership Questionnaire. Journal of Occupational and Organizational Psychology, 72(4), 441-462. DOI: https://doi.org/10.1348/096317999166789

Bagozzi, R. P.; Fornell, C. (1982). Theoretical concepts, measurement, and meaning. En C. Fornell (Ed.) A second generation of mulivariate analysis. (5-23). Santa Barbara: Praeger.

Bowersox, D. J.; Closs, D. J.; Stank, T. P.; Keller, S. B. (2000). How supply chain competency leads to business success. Supply Chain Management Review, 4(4), 70-78.

Boyer, K. K.; Lewis, M. W. (2002). Competitive priorities: investigating the need for trade-offs in operations strategy. Production and Operations Management, 11(1), 9-20. DOI: https://doi.org/10.1111/j.1937-5956.2002.tb00181.x

Bryant, S. E. (2003). The role of transformational and transactional leadership in creating, sharing and exploiting organizational knowledge. Journal of Leadership \& Organizational Studies, 9(4), 32-44. DOI: https://doi. org/10.1177/107179190300900403

Carmeli, A.; Gelbard, R.; Reiter-Palmon, R. (2013). Leadership, creative problem-solving capacity, and creative performance: the importance of knowledge sharing. Human Resource Management, 52(1), 95-121. DOI: https://doi.org/10.1002/ hrm.21514

Carrillo, J. (2014). ¿De qué maquila me hablas? Reflexiones sobre las complejidades de la industria maquiladora en México. Frontera Norte, 26(3), 75-98.

Chang, S.-C.; Yang, C.-L.; Cheng, H.-C.; Sheu, C. (2003). Manufacturing flexibility and business strategy: an empirical study of small and medium sized firms. International Journal of Production Economics, 83(1), 13-26. DOI: https://doi. org/10.1016/S0925-5273(02)00263-3

Chiang, M.; Méndez, G.; Sánchez, G. (2010). Cómo influye la satisfacción laboral sobre el desempeño: caso empresa de retail. Theoria, 19(2), 21-36.

Chin, W. W. (1998). Issues and Opinion on Structural Equation Modeling. MIS Quarterly, 22(1), 7-16.

Cubillos-Rivera, B.; Velásquez-Muriel, F. C.; Reyes-Nova, M. (2014). Plan de mejoramiento de variables del clima organizacional que afectan al desempeño laboral en una entidad del Estado. Suma de Negocios, 5(10), 69-73. DOI: https://doi.org/10.1016/S2215-910X(14)70012-6

de Leeuw, S.; van den Berg, J. (2011). Improving operational performance by influencing shopfloor behavior via performance management practices. Journal of Operations Management, 29(3), 224-235. DOI: https://doi. org/10.1016/j.jom.2010.12.009
Delgado-Almonte, M.; Pedraja-Rejas, L.; Rodríguez-Ponce, E. (2010). Estilos de liderazgo y gestión de conocimiento en pequeñas empresas. Técnica administrativa, 9(1), 1-13.

Durán, M. G.; Castañeda, D. I. (2015). Relación entre liderazgo transformacional y transaccional con la conducta de compartir conocimiento en dos empresas de servicios. Acta Colombiana de Psicología, 18(1), 135-147. DOI: https://doi. org/10.14718/ACP.2015.18.1.13

Fornell, C.; Bookstein, F. L. (1982). Two structural equation models: LISREL and PLS applied to consumer exit-voice theory. Journal of Marketing Research, 19(4), 440-452. DOI: https://doi.org/10.1177/002224378201900406

García-Guiu, C.; Moya-Morales, M.; Molero-Alonso, F.; MorianoLeón, J. A. (2016). Transformational leadership and group potency in small military units: The mediating role of group identification and cohesion. Revista de Psicología del Trabajo $y$ de las Organizaciones, 32(3), 145-152. DOI: https://doi. org/10.1016/j.rpto.2016.06.002

Gefen, D.; Straub, D. (2005). A practical guide to factorial validity using PLS-graph: tutorial and annotated example. Communications of the Association for Information Systems, 16, 91-109. DOI: https://doi.org/10.17705/1CAIS.01605

Gómez, D. (2006). Modelos para la creación y gestión del conocimiento: una aproximación teórica. Educar, 37, 25-39.

Hair, J. F.; Anderson, R. E.; Tatham, R. L.; Black, W. C. (2007). Análisis multivariante. Madrid: Pearson Prentice Hall.

Henseler, J.; Ringle, C. M.; Sarstedt, M. (2015). A new criterion for assessing discriminant validity in variance-based structural equation modeling. Journal of the Academy of Marketing Science, 43(1), 115-135. DOI: https://doi.org/10.1007/s11747-0140403-8

Herlina, E.; Syarifudin, D.; Kartika, R. (2019). The local knowledge transfer based on continuous improvement implementation at SMEs Group. Journal of Management Review, 3(1), 277-282. DOI: https://doi.org/10.25157/jmr. v3i1.1805

Hermosilla, D.; Amutio, A.; Costa, S., da; Páez, D. (2016). El Liderazgo transformacional en las organizaciones: variables mediadoras y consecuencias a largo plazo. Revista de Psicología del Trabajo y de las Organizaciones, 32(3), 135-143. DOI: https://doi.org/10.1016/j.rpto.2016.06.003

Hoch, J. E.; Bommer, W. H.; Dulebohn, J. H.; Wu, D. (2018). Do ethical, authentic, and servant leadership explain variance above and beyond transformational leadership? A metaanalysis. Journal of Management, 44(2), 501-529. DOI: https://doi.org/10.1177/0149206316665461

Hulland, J. (1999). Use of partial least squares (PLS) in strategic management research: a review of four recent studies. Strategic Management Journal, 20(2), 195204. DOI: https://doi.org/10.1002/(SICI)10970266(199902)20:2\%3C195::AID-SMJ13\%3E3.0.CO;2-7 
Inman, R.A.; Sale, R. S.; Green Jr., K.W.; Whitten, D. (2011). Agile manufacturing: relation to JIT, operational performance and firm performance. Journal of Operations Management, 29(4), 343-355. DOI: https://doi.org/10.1016/j.jom.2010.06.001

Jasimuddin, S. M.; Zhang, Z. (2009). The symbiosis mechanism for effective knowledge transfer. Journal of the Operational Research Society, 60(5), 706-716. DOI: https://doi. org/10.1057/palgrave.jors.2602613

Kane, A. A.; Argote, L.; Levine, J. M. (2005). Knowledge transfer between groups via personnel rotation: effects of social identity and knowledge quality. Organizational Behavior and Human Decision Processes, 96(1), 56-71. DOI: https://doi. org/10.1016/j.obhdp.2004.09.002

Machorro, F.; Mercado, P.; Cernas, D.; Romero, M. (2016). Influencia del capital relacional en el desempeño organizacional de las instituciones de educación superior tecnológica. Innovar Journal, 26(60), 35-50. DOI: https://doi. org/10.15446/innovar.v26n60.55531

Máynez, A. I. (2011). La transferencia de conocimiento organizacional como fuente de ventaja competitiva sostenible: modelo integrador de factores y estrategias. Puebla: Universidad Popular Autónoma del Estado de Puebla.

Menon, S. T. (2001). Employee empowerment: an integrative psychological approach. International Association for Applied Psychology, 50(1), 153-180. DOI: https://doi. org/10.1111/1464-0597.00052

Morales, V. J. G.; Martínez, M. M. R.; Montes, F. J. L. (2007). Liderazgo transformacional: influencia en la visión compartida, aprendizaje, innovación y resultado organizativo. Revista Europea de Dirección y Economía de la Empresa, 16(4), 24-46.

Oliva-Abusleme, M.; Molina-Fuentes, S. (2016). Estilos de liderazgo y satisfacción laboral en trabajadores de establecimientos de atención primaria en salud de la comuna de Chillán. Revista Academia \& Negocios, 2(1), 41-56. Recuperado de https://revistas.udec.cl/index.php/ran/ article/view/3021

Oviedo, H. C.; Campo-Arias, A. (2005). Aproximación al uso del coeficiente alfa de Cronbach. Revista Colombiana de Psiquiatría, 34(4), 572-580. DOI: https://doi.org/10.7705/ biomedica.v26i4.327

Paz, J. (2017). Transferencia del conocimiento como agente articulado de la competitividad en el sector bancario. Telos. Revista de estudios interdisciplinarios en ciencias sociales, 19(3), 408-430. Recuperado de http://ojs.urbe.edu/index.php/ telos/article/view/724/654

Song, J.; Kolb, J. A.; Lee, U.; Kim, H. (2012). Role of transformational leadership in effective organizational knowledge creation practices: mediating effects of employees' work engagement. Human Resource Development Quarterly, 23(1), 65-101. DOI: https://doi.org/10.1002/hrdq.21120
Srivastava, A.; Bartol, K. M.; Locke, E. A. (2006). Empowering leadership in management teams: effects on knowledge sharing, efficacy, and performance. Academy of Management Journal, 49(6), 1239-1251. DOI: https://doi.org/10.5465/ amj.2006.23478718

Tsai, W. (2001). Knowledge transfer in intraorganizational networks: effects of network position and absorptive capacity on business unit innovation and performance. Academy of Management Journal, 44(5), 996-1004. DOI: https://doi. org $/ 10.5465 / 3069443$

Uribe, A. F.; Molina, J. M.; Torres, F. V. C.; Barbosa, D.; Espinosa, J. C. (2013). Liderar equipos de alto desempeño: un gran reto para las organizaciones actuales. Universidad \& Empresa, 15(25), 53-71. Recuperado de https://bit.ly/3sDWcFT

Vargas-Salgado. M.; Máynez-Guaderrama. A.; Cavazos-Arroyo. J.; Cervantes-Benavides. L. (2016). Validez de contenido de un instrumento de medición para medir el Liderazgo Transformacional. Revista Global de Negocios, 4(1), 35-45.

Villa, O.; Márquez, B. L. (2016). Relación del empowerment con el compromiso organizacional influenciado por el capital humano y prácticas de alto involucramiento de empleados en maquiladoras automotrices. Global Conference on Business \& Finance Proceedings, 11(1), 855-867.

Wang, E. T.; Chia-Lin, C.; Jiang, J. J.; Klein, G. (2007). Improving enterprise resource planning - ERP - fit to organizational process through knowledge transfer. International Journal of Information Management, 27(3), 200-212. DOI: https://doi. org/10.1016/j.ijinfomgt.2007.02.002

Ward, P. T.; Duray, R. (2000). Manufacturing strategy in context: environment, competitive strategy and manufacturing strategy. Journal of Operations Management, 18(2), 123-138. DOI: https://doi.org/10.1016/S0272-6963(99)00021-2

Ynzunza, C.; Izar, J. (2013). Estrategia, orientación al mercado y desempeño organizacional. Ciencia Tecnológica, (45), 5-11. 